\title{
Correction: Synaptic phospholipids as a new target for cortical hyperexcitability and E/I balance in psychiatric disorders
}

\author{
Carine Thalman ${ }^{1} \cdot$ Guilherme Horta $^{2} \cdot$ Lianyong Qiao $^{2} \cdot$ Heiko Endle $^{2} \cdot$ Irmgard Tegeder $^{3} \cdot$ Hong Cheng $^{2} \cdot$ \\ Gregor Laube $^{4}$ - Torfi Sigurdsson ${ }^{5}$ Maria Jelena Hauser ${ }^{5}$ - Stefan Tenzer $\mathbb{1}^{6}$. Ute Distler ${ }^{6,7}$ - Junken Aoki ${ }^{8}$. \\ Andrew J. Morris ${ }^{9} \cdot$ Gerd Geisslinger $^{3}$ - Jochen Röper ${ }^{5} \cdot$ Sergei Kirischuk $^{10} \cdot$ Heiko J. Luhmann ${ }^{10}$. \\ Konstantin Radyushkin ${ }^{7}$ Robert Nitsch ${ }^{11} \cdot$ Johannes Vogt ${ }^{2}$
}

Published online: 2 January 2019

(c) The Author(s) 2018

\section{Correction to: Molecular Psychiatry}

https://doi.org/10.1038/s41380-018-0053-1;

published online 9 May 2018.

Following the publication of this article the authors noted that Torfi Sigurdsson's name was misspelled. Instead of
Sigrudsson it should be Sigurdsson. The PDF and HTML versions of the paper have been modified accordingly.

The authors would like to apologise for this error and the inconvenience this may have caused.
These authors contributed equally: Carine Thalman, Guilherme Horta, Lianyong Qiao.

These authors jointly supervised: Robert Nitsch, Johannes Vogt.

The original article can be found online at https://doi.org/10.1038/ s41380-018-0053-1.

Robert Nitsch

nitschr@uni-muenster.de

$\triangle$ Johannes Vogt

johannes.vogt@unimedizin-mainz.de

1 Department of Neurology, University Medical Center, Johannes Gutenberg-University, Mainz, Germany

2 Institute for Microscopic Anatomy and Neurobiology, University Medical Center, Johannes Gutenberg-University, Mainz, Germany

3 Institute of Clinical Pharmacology, Goethe University Frankfurt, Frankfurt, Germany

4 Institute for Integrative Neuroanatomy, Charité Universitätsmedizin, Berlin, Germany

5 Institute of Neurophysiology, Neuroscience Center, Goethe
University Frankfurt, Frankfurt, Germany

6 Institute for Immunology, University Medical Center, Johannes Gutenberg-University, Mainz, Germany

7 Focus Program Translational Neuroscience, Johannes GutenbergUniversity, Mainz, Germany

8 Graduate School of Pharmaceutical Sciences, Tohoku University, Aoba-ku, Sendai, Japan

9 Division of Cardiovascular Medicine, Gill Heart Institute, University of Kentucky, Lexington, KY, USA

10 Institute of Physiology, University Medical Center, Johannes Gutenberg-University, Mainz, Germany

11 Institute for Translational Neuroscience, University Medical Center, Westfälische Wilhems-University Münster, AlbertSchweitzer-Campus, Münster, Germany 